\title{
On Borel Equivalence Relations Related To Self-Adjoint Operators
}

\author{
Hiroshi Ando@ Yasumichi Matsuzawa
}

August 17, 2018

\begin{abstract}
In a recent work, we initiated the study of Borel equivalence relations defined on the Polish space $\mathrm{SA}(H)$ of self-adjoint operators on a Hilbert space $H$, focusing on the difference between bounded and unbounded operators. In this paper, we extend the analysis and show how the difficulty of specifying the domains of self-adjoint operators is reflected in Borel complexity of associated equivalence relations. More precisely, we show that the equality of domains, regarded as an equivalence relation on $\mathrm{SA}(H)$ is continously bireducible with the orbit equivalence relation of the standard Borel group $\ell^{\infty}(\mathbb{N})$ on $\mathbb{R}^{\mathbb{N}}$. Moreover, we show that generic self-adjoint operators have purely singular continuous spectrum equal to $\mathbb{R}$.
\end{abstract}

2010 AMS subject classification: Primary 03E15, Secondary: 34L05

Keywords: Unbounded self-adjoint operators, Borel equivalence relations

\section{Introduction}

In the recent paper [AM14, the authors have studied Borel complexity of various equivalence relations defined on the space $\mathrm{SA}(H)$ of all (not necessarily bounded) self-adjoint operators on a separable and infinite-dimensional Hilbert space $H$ equipped with the strong resolvent topology (SRT). One major difference between bounded and unbounded operators is that due to the domain problems, $\mathrm{SA}(H)$ is not even a vector space: recall that the sum of selfadjoint operators $A, B$ is defined as the operator $C$ with $\operatorname{dom}(C)=\operatorname{dom}(A) \cap \operatorname{dom}(B)$ and $C \xi:=A \xi+B \xi, \xi \in \operatorname{dom}(C)$. In general, there is no reason to expect that $C$ is densely defined even if $\operatorname{dom}(A), \operatorname{dom}(B)$ are dense. In fact, Israel [Isr04 has shown that if $A \in \mathrm{SA}(H)$ has empty essential spectrum, then the set of all unitaries $u$ satisfying $\operatorname{dom}(A) \cap u \cdot \operatorname{dom}(A)=\{0\}$ forms a norm dense $G_{\delta}$ subset of the unitary group $\mathcal{U}(H)$. Thus $\operatorname{dom}\left(A+u A u^{*}\right)=\{0\}$ for norm-generic $u$. Therefore, it is natural to expect that the domain equivalence relation

$$
A E_{\mathrm{dom}}^{\mathrm{SA}(H)} B \Leftrightarrow \operatorname{dom}(A)=\operatorname{dom}(B)
$$

has a high degree of complexity. In this paper, we determine its exact Borel complexity by showing that $E_{\mathrm{dom}}^{\mathrm{SA}(H)}$ is an $F_{\sigma}$ (but not $K_{\sigma}$ ) equivalence relation, and that it is continuously bireducible (see $\S 2$ for the definition) with the $\ell^{\infty}(\mathbb{N}, \mathbb{R})$-orbit equivalence relation $E_{\ell^{\infty}}^{\mathbb{R}^{\mathbb{N}}}$ defined on $\mathbb{R}^{\mathbb{N}}$ by

$$
\left(a_{n}\right)_{n=1}^{\infty} E_{\ell^{\infty}}^{\mathbb{R}^{\mathbb{N}}}\left(b_{n}\right)_{n=1}^{\infty} \Leftrightarrow \sup _{n \in \mathbb{N}}\left|a_{n}-b_{n}\right|<\infty, \quad\left(a_{n}\right)_{n=1}^{\infty},\left(b_{n}\right)_{n=1}^{\infty} \in \mathbb{R}^{\mathbb{N}}
$$


Since Rosendal [Ros05, Proposition 19] has shown that $E_{\ell^{\mathbb{N}}}^{\mathbb{R}^{\mathbb{N}}}$ is universal for $K_{\sigma}$-equivalence relations, $E_{\mathrm{dom}}^{\mathrm{SA}(H)}$ also enjoys this property. Moreover, since by this universality the notorious $K_{\sigma}$ equivalence relation $E_{1}$ (see $\S 3$ ) is Borel reducible to $E_{\mathrm{dom}}^{\mathrm{SA}(H)}, E_{\mathrm{dom}}^{\mathrm{SA}(H)}$ is not Borel reducible to any orbit equivalence relation of a Borel action of a Polish group, by the Kechris-Louveau Theorem [KL97, Theorem 4.2]. Moreover, we show that the related equivalence relation $E_{\mathrm{dom}, u}^{\mathrm{SA}(H)}$ (unitary equivalence of domains) given by

$$
A E_{\mathrm{dom}, u}^{\mathrm{SA}(H)} B \Leftrightarrow \exists u \text { unitary }[u \cdot \operatorname{dom}(A)=\operatorname{dom}(B)]
$$

is Borel reducible to a $K_{\sigma}$ equivalence relation, whence $E_{\mathrm{dom}, u}^{\mathrm{SA}(H)} \leq_{B} E_{\mathrm{dom}}^{\mathrm{SA}(H)}$ as a corollary. Finally, we strengthen our previous genericity result [AM14, Theorem 3.17 (1)] that elements in $\mathrm{SA}(H)$ which have essential spectrum $\mathbb{R}$, form a dense $G_{\delta}$ set. Namely we prove that elements in $\mathrm{SA}(H)$ which have purely singular continuous spectrum $\mathbb{R}$, forms a dense $G_{\delta}$ set in $\mathrm{SA}(H)$. This shows that although every self-adjoint operator can be approximated by diagonal operators (Weyl-von Neumann Theorem), generic self-adjoint operators have rather pathological spectral properties (cf. CN98, LPS05]). The proof is based on Simon's Wonderland Theorem Sim95].

\section{Preliminaries}

We refer the reader to [AM14, §2] for relevant definitions and notation. Basic facts about operator theory (resp. descriptive set theory) can be found in Sch10 (resp. in Gao09, Hjo00, Kec96]). Below we give some definitions here for convenience. Let $H$ be a separable infinite-dimensional Hilbert space.

Definition 2.1. The strong resolvent topology (SRT) on the space $\mathrm{SA}(H)$ of all self-adjoint operators on $H$ is the coarsest topology which makes the map $\mathrm{SA}(H) \ni A \mapsto(A-i)^{-1} \in \mathbb{B}(H)$ continuous with respect to the strong operator topology (SOT).

$\mathrm{SA}(H)$ is Polish with respect to $\mathrm{SRT}$. The domain of $A \in \mathrm{SA}(H)$ is written as $\operatorname{dom}(A)$.

Definition 2.2. Let $E$ (resp. $F$ ) be equivalence relations on a Polish space $X$ (resp. $Y$ ). We say that $E$ is Borel (resp. continuously) reducible to $F$, denoted $E \leq_{B} F$ (resp. $E \leq_{c} F$ ), if there is a Borel (resp. continuous) map $f: X \rightarrow Y$ which is a reduction of $E$ to $F$ (i.e., $x E y \Leftrightarrow f(x) F f(y)$ holds for $x, y \in X)$. If moreover $f$ is injective, we say that $E$ is Borel (resp. continuously) embeddable into $F$, denoted $E \sqsubseteq_{B} F$ (resp. $E \sqsubseteq_{c} F$ ). We say that $E$ is Borel (resp. continuously) bireducible with $F$, if $E \leq_{B} F$ and $F \leq_{B} E$ (resp. $E \leq_{c} F$ and $F \leq_{c} E$ ) hold. In this case we write $E \sim_{B} F$ (resp. $E \sim_{c} F$ ).

In the next section we consider the following three equivalence relations.

Definition 2.3. We define $E_{\ell^{\infty}}^{\mathbb{R}^{\mathbb{N}}}, E_{\mathrm{dom}}^{\mathrm{SA}(H)}$ and $E_{\mathrm{dom}, u}^{\mathrm{SA}(H)}$ by:

(1) The equivalence relation $E_{\ell \infty}^{\mathbb{R}^{\mathbb{N}}}$ on the Polish space $\mathbb{R}^{\mathbb{N}}$ is the orbit equivalence relation of the action of the standard Borel group $\ell^{\infty}=\ell^{\infty}(\mathbb{N})$ on $\mathbb{R}^{\mathbb{N}}$ by addition. In other words, we have $\left(a_{n}\right)_{n=1}^{\infty} E_{\ell \infty}^{\mathbb{R}^{\mathbb{N}}}\left(b_{n}\right)_{n=1}^{\infty} \Leftrightarrow \sup _{n \in \mathbb{N}}\left|a_{n}-b_{n}\right|<\infty$ for $\left(a_{n}\right)_{n=1}^{\infty},\left(b_{n}\right)_{n=1}^{\infty} \in \mathbb{R}^{\mathbb{N}}$.

(2) The equivalence relation $E_{\mathrm{dom}}^{\mathrm{SA}(H)}$ on $\mathrm{SA}(H)$ is given by $A E_{\operatorname{dom}}^{\mathrm{SA}(H)} B \Leftrightarrow \operatorname{dom}(A)=$ $\operatorname{dom}(B)$. 
(3) The equivalence relation $E_{\mathrm{dom}, u}^{\mathrm{SA}(H)}$ on $\mathrm{SA}(H)$ is given by $A E_{\mathrm{dom}, u}^{\mathrm{SA}(H)} B \Leftrightarrow \exists u \in \mathcal{U}(H)[u$. $\operatorname{dom}(A)=\operatorname{dom}(B)]$.

We also recall a result on operator ranges. Recall that a subspace $\mathcal{R} \subset H$ is an operator range in $H$, if $\mathcal{R}$ is equal to the range $\operatorname{Ran}(T)$ for some $T \in \mathbb{B}(H)$. We may choose $T$ to be self-adjoint with $0 \leq T \leq 1$. In this case, we set $H_{n}:=E_{T}\left(\left(2^{-n-1}, 2^{-n}\right]\right) H(n=0,1, \cdots)$. Then $H_{n}$ are mutually orthogonal closed subspaces of $H$ with $H=\bigoplus_{n=0}^{\infty} H_{n}$ (by the density of $\mathcal{R}$ ). $\left\{H_{n}\right\}_{n=0}^{\infty}$ are called the associated subspaces for $T$ (see [FW71, $\left.\S 3\right]$ for details). Since we are only concerned with dense operator ranges, we state the following result [FW71, Theorem 3.3] for dense operator ranges (in this case the condition (1) of the cited theorem is automatic).

Theorem 2.4 (Köthe, Fillmore-Williams). Let $\mathcal{R}$ and $\mathcal{S}$ be dense operator ranges in $H$ with associated subspaces $\left\{H_{n}\right\}_{n=0}^{\infty}$ and $\left\{K_{n}\right\}_{n=0}^{\infty}$, respectively. Then there exists $u \in \mathcal{U}(H)$ such that $u \mathcal{R}=\mathcal{S}$, if and only if there exists $k \geq 0$ such that for each $n \geq 0$ and $l \geq 0$, one has

$$
\begin{aligned}
& \operatorname{dim}\left(H_{n} \oplus \cdots \oplus H_{n+l}\right) \leq \operatorname{dim}\left(K_{n-k} \oplus \cdots \oplus K_{n+l+k}\right) \\
& \operatorname{dim}\left(K_{n} \oplus \cdots \oplus K_{n+l}\right) \leq \operatorname{dim}\left(H_{n-k} \oplus \cdots \oplus H_{n+l+k}\right),
\end{aligned}
$$

where we use the convention $H_{m}=K_{m}=\{0\}$ for $m<0$.

Finally, for $A \in \mathrm{SA}(H)$, we denote by $\sigma_{\mathrm{p}}(A), \sigma_{\mathrm{ac}}(A)$ and $\sigma_{\mathrm{sc}}(A)$ the set of eigenvalues, absolutely continuous spectrum, and singular continuous spectrum of $A$, respectively (see [RS81, §VII.2]). We put $\sigma_{\mathrm{ac}}(A)=\emptyset\left(\right.$ resp. $\left.\sigma_{\mathrm{sc}}(A)=\emptyset\right)$ if there is no absolutely continuous part (resp. singular continuous part) of $A$, and we say that $A$ has purely singular continuous spectrum, if $\sigma_{\mathrm{p}}(A)=\emptyset=\sigma_{\mathrm{ac}}(A)$ holds.

\section{Main Results}

Now we state the main result.

Theorem 3.1. $E_{\mathrm{dom}}^{\mathrm{SA}(H)}$ is an $F_{\sigma}$ equivalence relation which is continuously bireducible with $E_{\ell^{\infty}}^{\mathbb{R}^{\mathbb{N}}}$

Before going to the proof, let us state an immediate corollary. We need two important results. Recall that a subspace of a topological space is called $K_{\sigma}$ or $\sigma$-compact, if it is a countable union of compact subsets. First, Rosendal [Ros05, Proposition 19] has shown that

Theorem 3.2 (Rosendal). $E_{\ell^{\infty}}^{\mathbb{R}^{\mathbb{N}}}$ is universal for $K_{\sigma}$ equivalence relations in the sense that any $K_{\sigma}$ equivalence relation on a Polish space is Borel reducible to $E_{\ell^{\infty}}^{\mathbb{R}^{\mathbb{N}}}$.

Secondly, recall the $K_{\sigma}$ equivalence relation $E_{1}$ on $\mathcal{C}^{\mathbb{N}}$ (where $\mathcal{C}=2^{\mathbb{N}}$ ) defined by

$$
\left(a_{n}\right)_{n=1}^{\infty} E_{1}\left(b_{n}\right)_{n=1}^{\infty} \Leftrightarrow \exists N \in \mathbb{N} \forall n \geq N\left[a_{n}=b_{n}\right] .
$$

Since $\mathcal{C}$ and $\mathbb{R}$ are Borel isomorphic, $E_{1}$ may alternatively be defined (when talking about Borel reducibility) as the tail equivalence relation on $\mathbb{R}^{\mathbb{N}}$. Kechris-Louveau KL97, Theorem 4.2] has shown that $E_{1}$ is an obstruction for a given equivalence relation to be Borel reducible to orbit equivalence:

Theorem 3.3 (Kechris-Louveau). $E_{1} \mathbb{Z}_{B} E_{G}^{X}$ for any Polish group $G$ and Polish $G$-space $X$. 
Here, $E_{G}^{X}$ stands for the orbit equivalence relation associated with the Borel $G$-action. Since there are many orbit equivalence relations that are turbulent (in the sense of [Hjo00]) and Borel reducible to $E_{\ell^{\infty}}^{\mathbb{R}^{\mathbb{N}}}$ (e.g. $\ell^{p}(\mathbb{N})(1 \leq p<\infty)$ actions on $\mathbb{R}^{\mathbb{N}}$ ), Theorems 3.1, 3.2 and 3.3 imply that:

Corollary 3.4. $E_{\mathrm{dom}}^{\mathrm{SA}(H)}$ is universal for $K_{\sigma}$-equivalence relations. In particular, it is unclassifiable by countable structures, not Borel reducible to orbit equivalence relation of any Polish group action.

Now we prove Theorem 3.1 in few steps.

Proposition 3.5. $E_{\mathrm{dom}}^{\mathrm{SA}(H)}$ is an $F_{\sigma}$ equivalence relation which is not $K_{\sigma}$.

The proof relies on Douglas' range inclusion Theorem [Dou66] (cf. [FW71, Theorem 2.1]).

Theorem 3.6 (Douglas). Let $A, B \in \mathbb{B}(H)$. Then $\operatorname{Ran}(A) \subset \operatorname{Ran}(B)$ holds if and only if there exists $\lambda>0$ such that $A A^{*} \leq \lambda B B^{*}$.

Proof of Proposition 3.5. It is clear that $\tau: \mathrm{SA}(H)^{2} \ni(A, B) \mapsto(B, A) \in \mathrm{SA}(H)^{2}$ is a homeomorphism. Define $\mathcal{S}:=\left\{(A, B) \in \mathrm{SA}(H)^{2} ; \operatorname{dom}(A) \subset \operatorname{dom}(B)\right\}$. Since $E_{\operatorname{dom}}^{\mathrm{SA}(H)}=\mathcal{S} \cap \tau(\mathcal{S})$, it suffices to show that $\mathcal{S}$ is $F_{\sigma}$ in $\mathrm{SA}(H)^{2}$. For $A, B \in \mathrm{SA}(H)$, we have $\operatorname{dom}(A)=$ $\operatorname{Ran}\left((|A|+1)^{-1}\right), \operatorname{dom}(B)=\operatorname{Ran}\left((|B|+1)^{-1}\right)$. Therefore Theorem 3.6 shows that

$$
\begin{aligned}
\operatorname{dom}(A) \subset \operatorname{dom}(B) & \Leftrightarrow \exists \lambda>0\left[(|A|+1)^{-2} \leq \lambda(|B|+1)^{-2}\right] \\
& \Leftrightarrow \exists k \in \mathbb{N} \forall \xi \in H\left[\left\langle\xi,(|A|+1)^{-2} \xi\right\rangle \leq k\left\langle\xi,(|B|+1)^{-2} \xi\right\rangle\right] .
\end{aligned}
$$

Therefore $\mathcal{S}=\bigcup_{k \in \mathbb{N}} \bigcap_{\xi \in H} S_{k, \xi}$, where $S_{k, \xi}:=\left\{(A, B) ;\left\langle\xi,(|A|+1)^{-2} \xi\right\rangle \leq k\left\langle\xi,(|B|+1)^{-2} \xi\right\rangle\right\}$. It is easy to see that $\mathrm{SA}(H) \ni A \mapsto(|A|+1)^{-2} \in \mathbb{B}(H)$ is SRT-SOT continuous, hence each $S_{k, \xi}$ is SRT-closed. Therefore $\mathcal{S}$ is $F_{\sigma}$. The last assertion follows from the fact that $\operatorname{SA}(H)$ is not $K_{\sigma}$ (it contains a homeomorphic copy of $\mathbb{R}^{\mathbb{N}}$ ) and a well-known fact: note that if an equivalence relation $E$ on a Polish space $X$ is $K_{\sigma}$, then $X$ must be $K_{\sigma}$.

Proof of Theorem 3.1. $E_{\mathrm{dom}}^{\mathrm{SA}(H)}$ is $F_{\sigma}$ but not $K_{\sigma}$ by Proposition 3.5. We show that $E_{\mathrm{dom}}^{\mathrm{SA}(H)}$ is continuously bireducible with $E_{\ell^{\infty}}^{\mathbb{R}^{\mathbb{N}}}$. We first show that $E_{\text {dom }}^{\mathrm{SA}(H)} \leq_{c} E_{\ell \infty}^{\mathbb{R}^{\mathbb{N}}}$. Fix a dense countable subset $\left\{\xi_{n}\right\}_{n=1}^{\infty}$ of $H$. Given $A \in \mathrm{SA}(H)$, define $T_{A}:=(|A|+1)^{-2}$. Since $T_{A}$ is positive and 0 is not an eigenvalue for $T_{A},\left\langle\xi_{n}, T_{A} \xi_{n}\right\rangle>0$ for every $n \in \mathbb{N}$. Moreover, $A \mapsto T_{A}$ is SRT-SOT continuous by functional calculus. Therefore we may define a continuous map $\varphi: \mathrm{SA}(H) \rightarrow \mathbb{R}^{\mathbb{N}}$ by

$$
\varphi(A):=\left(a_{n}(A)\right)_{n=1}^{\infty}, \quad a_{n}(A):=\log \left(\left\langle\xi_{n}, T_{A} \xi_{n}\right\rangle\right), \quad A \in \mathrm{SA}(H), n \in \mathbb{N} .
$$

We show that $\varphi$ is a reduction map. Let $A, B \in \mathrm{SA}(H)$. By the proof of Proposition 3.5, we have

$$
\begin{aligned}
\operatorname{dom}(A)=\operatorname{dom}(B) & \Leftrightarrow \exists C_{1}>0 \exists C_{2}>0\left[C_{1} T_{B} \leq T_{A} \leq C_{2} T_{B}\right] \\
& \Leftrightarrow \exists C_{1}>0 \exists C_{2}>0 \forall n \in \mathbb{N}\left[C_{1}\left\langle\xi_{n}, T_{B} \xi_{n}\right\rangle \leq\left\langle\xi_{n}, T_{A} \xi_{n}\right\rangle \leq C_{2}\left\langle\xi_{n}, T_{B} \xi_{n}\right\rangle\right] \\
& \Leftrightarrow \exists C_{1}>0 \exists C_{2}>0 \forall n \in \mathbb{N}\left[\log C_{1} \leq a_{n}(A)-a_{n}(B) \leq \log C_{2}\right] \\
& \Leftrightarrow \sup _{n \in \mathbb{N}}\left|a_{n}(A)-a_{n}(B)\right|<\infty \\
& \Leftrightarrow \varphi(A) E_{\ell^{\infty}}^{\mathbb{R}^{\mathbb{N}}} \varphi(B),
\end{aligned}
$$


which shows that $E_{\mathrm{dom}}^{\mathrm{SA}(H)} \leq_{c} E_{\ell^{\infty}}^{\mathbb{R}^{\mathbb{N}}}$.

Next we show that $E_{\ell^{\infty}}^{\mathbb{R}^{\mathbb{N}}} \leq_{c} E_{\text {dom }}^{\mathrm{SA}(H)}$. The proof is similar to the first part. Fix a complete orthonormal system (CONS) $\left\{\eta_{n}\right\}_{n=1}^{\infty}$ for $H$. For each $\left(x_{n}\right)_{n=1}^{\infty} \in \mathbb{R}^{\mathbb{N}}$, define $\left(\tilde{x}_{n}\right)_{n=1}^{\infty} \in \mathbb{R}_{\geq 0}^{\mathbb{N}}$ by

$$
\left(\tilde{x}_{2 n-1}, \tilde{x}_{2 n}\right)=\left\{\begin{array}{ll}
\left(\left|x_{n}\right|, 0\right) & \left(x_{n} \geq 0\right) \\
\left(0,\left|x_{n}\right|\right) & \left(x_{n}<0\right)
\end{array}, \quad n \in \mathbb{N} .\right.
$$

Thus $\left(1,-\frac{1}{2}, 4,0, \cdots\right)$ is mapped to $\left(1,0,0, \frac{1}{2}, 4,0,0,0, \cdots\right)$, etc. It is easy to see that $\mathbb{R}^{\mathbb{N}} \ni$ $\left(x_{n}\right)_{n=1}^{\infty} \mapsto\left(\tilde{x}_{n}\right) \in \mathbb{R}_{>0}^{\mathbb{N}}$ is an injective continuous map satisfying

$$
\sup _{n \in \mathbb{N}}\left|x_{n}-y_{n}\right|<\infty \Leftrightarrow \sup _{n \in \mathbb{N}}\left|\tilde{x}_{n}-\tilde{y}_{n}\right|<\infty, \quad\left(x_{n}\right)_{n=1}^{\infty},\left(y_{n}\right)_{n=1}^{\infty} \in \mathbb{R}^{\mathbb{N}} .
$$

We define $\psi: \mathbb{R}^{\mathbb{N}} \rightarrow \mathrm{SA}(H)$ by

$$
\psi(\alpha):=\sum_{n=1}^{\infty}\left\{\exp \left(\frac{1}{2} \tilde{x}_{n}\right)-1\right\}\left\langle\eta_{n}, \cdot\right\rangle \eta_{n}, \quad \alpha=\left(x_{n}\right)_{n=1}^{\infty} \in \mathbb{R} .
$$

It is easy to see that $\psi$ is continuous, and

$$
T_{\psi(\alpha)}=(\psi(\alpha)+1)^{-2}=\sum_{n=1}^{\infty} \exp \left(-\tilde{x}_{n}\right)\left\langle\eta_{n}, \cdot\right\rangle \eta_{n}, \quad \alpha=\left(x_{n}\right)_{n=1}^{\infty} \in \mathbb{R}^{\mathbb{N}}
$$

We show that $\psi$ is a reduction map. Given $\alpha=\left(x_{n}\right)_{n=1}^{\infty}, \beta=\left(y_{n}\right)_{n=1}^{\infty} \in \mathbb{R}^{\mathbb{N}}$, we have (by (1))

$$
\begin{aligned}
\operatorname{dom}(\psi(\alpha))=\operatorname{dom}(\psi(\beta)) \Leftrightarrow & \exists C_{1}>0 \exists C_{2}>0\left[C_{1} T_{\psi(\beta)} \leq T_{\psi(\alpha)} \leq C_{2} T_{\psi(\beta)}\right] \\
& \Leftrightarrow \exists C_{1}>0 \exists C_{2}>0 \forall n \in \mathbb{N} \\
& \quad \quad \quad\left[C_{1} \exp \left(-\tilde{y}_{n}\right) \leq \exp \left(-\tilde{x}_{n}\right) \leq C_{2} \exp \left(-\tilde{y}_{n}\right)\right] \\
& \sup _{n \in \mathbb{N}}\left|\tilde{y}_{n}-\tilde{x}_{n}\right|<\infty \\
& \Leftrightarrow \alpha E_{\ell^{\infty}}^{\mathbb{R}^{\mathbb{N}}} \beta,
\end{aligned}
$$

whence $E_{\ell^{\infty}}^{\mathbb{R}^{\mathbb{N}}} \leq_{c} E_{\mathrm{dom}}^{\mathrm{SA}(H)}$. This shows that $E_{\ell^{\infty}}^{\mathbb{R}^{\mathbb{N}}}$ is continuously bireducible with $E_{\mathrm{dom}}^{\mathrm{SA}(H)}$.

As another corollary to Theorem 3.1, we prove that $E_{\mathrm{dom}, u}^{\mathrm{SA}(H)} \leq_{B} E_{\mathrm{dom}}^{\mathrm{SA}(H)}$. This is done by showing that $E_{\mathrm{dom}, u}^{\mathrm{SA}(H)}$ is Borel reducible to a $K_{\sigma}$ equivalence relation. Regard $\mathbb{N}^{*}:=\mathbb{N} \cup\{\infty\}$ as a one-point compactification of $\mathbb{N}=\{1,2, \cdots\}$. Thus $\mathbb{N}^{*}$ is homeomorphic to $\left\{\frac{1}{n} ; n \in \mathbb{N}\right\} \cup\{0\}$ by $n \mapsto \frac{1}{n}(n \in \mathbb{N})$ and $\infty \mapsto 0$. Consider the compact Polish space $X:=\prod_{n=0}^{\infty}\left(\mathbb{N}^{*} \cup\{0\}\right)$, and define $X_{0}:=\left\{\left(a_{n}\right)_{n=0}^{\infty} \in X ; \sum_{n=0}^{\infty} a_{n}=\infty\right\}$. Then $X_{0}$ is a (dense) $G_{\delta}$ subspace of $X$, whence Polish.

Definition 3.7. Define an equivalence relation $E_{\Sigma}$ on $X$ by $\left(a_{n}\right)_{n=0}^{\infty} E_{\Sigma}\left(b_{n}\right)_{n=0}^{\infty}$ if and only if there exists $k \geq 0$ such that for each $l \geq 0$ and $n \geq 0$,

$$
\sum_{i=0}^{l} a_{n+i} \leq \sum_{j=-k}^{l+k} b_{n+j} \text { and } \sum_{i=0}^{l} b_{n+i} \leq \sum_{j=-k}^{l+k} a_{n+j} .
$$

Here, we regard $a_{n}=b_{n}=0(n<0)$ and $\infty+n=n+\infty=\infty+\infty=\infty(n \in \mathbb{N})$. 
Proposition 3.8. $E_{\Sigma}$ is a $K_{\sigma}$ equivalence relation, and $\left.E_{\mathrm{dom}, u}^{\mathrm{SA}(H)} \sim_{B} E_{\Sigma}\right|_{X_{0}}\left(\leq_{B} E_{\Sigma}\right)$. In particular, $E_{\mathrm{dom}, u}^{\mathrm{SA}(H)}$ is Borel reducible to a $K_{\sigma}$ equivalence relation.

We omit the proof of the next easy lemma.

Lemma 3.9. For $n, m \in \mathbb{N} \cup\{0\}(n \leq m)$, the $\operatorname{map} X \ni\left(a_{k}\right)_{k=0}^{\infty} \mapsto \sum_{k=n}^{m} a_{k} \in \mathbb{N}^{*}$ is continuous.

Lemma 3.10. Let $a, b \in \mathbb{R}, a<b$, and let $I=(a, b),[a, b)$ or $(a, b]$. Then the map $\mathrm{SA}(H) \ni$ $A \mapsto \operatorname{rank}\left(E_{A}(I)\right) \in \mathbb{N}^{*}$ is Borel.

Proof. We show the case of $I=[a, b)$. Let $S_{n}:=\left\{A \in \mathrm{SA}(H) ; \operatorname{rank}\left(E_{A}([a, b))\right) \leq n\right\}(n \in \mathbb{N} \cup$ $\{0\}), S_{\infty}:=\left\{A \in \mathrm{SA}(H) ; \operatorname{rank}\left(E_{A}([a, b))\right)=\infty\right\}$. Then by a similar argument to the proof of [AM14, Proposition 3.18] (especially that $S_{n, k}$ defined there is SRT-closed), it can be shown that $S_{n}$ is SRT-closed. Therefore $\left\{A \in \mathrm{SA}(H) ; \operatorname{rank}\left(E_{A}([a, b))\right)=n\right\}=S_{n} \backslash S_{n-1}(n \geq 1)$ and $S_{0}$ are Borel. Then $S_{\infty}=\mathrm{SA}(H) \backslash \bigcup_{n \geq 0} S_{n}$ is Borel too. Thus the map $A \mapsto \operatorname{rank}\left(E_{A}(I)\right)$ is Borel.

Proof of Proposition [3.8. It is easy to see that $\operatorname{dom}(A)=\operatorname{dom}(|A|+1)$ for every $A \in \mathrm{SA}(H)$, and $\operatorname{dom}(A)=\operatorname{Ran}\left((|A|+1)^{-1}\right)$. The associated subspaces for $T_{A}=(|A|+1)^{-1}$ are

$$
H_{n}\left(T_{A}\right)=E_{T_{A}}\left(\left(2^{-n-1}, 2^{n}\right]\right) H, \quad n \geq 0 .
$$

Note that for $\lambda \in \sigma(A)$,

$$
(|\lambda|+1)^{-1} \in\left(2^{-n-1}, 2^{n}\right] \Leftrightarrow \lambda \in \underbrace{\left(1-2^{n+1}, 1-2^{n}\right] \cup\left[2^{n}-1,2^{n+1}-1\right)}_{=: I_{n} \cup J_{n}} .
$$

Let $d_{0}(A):=\operatorname{rank}\left(E_{A}(-1,1)\right)$ and $d_{n}(A):=\operatorname{dim} H_{n}\left(T_{A}\right)=\operatorname{rank}\left(E_{A}\left(I_{n}\right)\right)+\operatorname{rank}\left(E_{A}\left(J_{n}\right)\right)(n \geq$ 1 ). By Lemma 3.10, $d_{n}: \mathrm{SA}(H) \rightarrow \mathbb{N}^{*}$ is Borel for each $n \geq 0$.

Now, note that $E_{\Sigma}=\bigcup_{k=0}^{\infty} E_{k}$, where

$$
E_{k}:=\bigcap_{l, n=0}^{\infty}\left\{\left(\left(a_{n}\right)_{n=0}^{\infty},\left(b_{n}\right)_{n=0}^{\infty}\right) ; \sum_{i=0}^{l} a_{n+i} \leq \sum_{j=-k}^{l+k} b_{n+j} \text { and } \sum_{i=0}^{l} b_{n+i} \leq \sum_{j=-k}^{l+k} a_{n+j}\right\}
$$

It is immediate to see that $E_{\Sigma}$ is $K_{\sigma}$ because each $E_{k}$ is a closed subset of the compact space $X \times X$ by Lemma 3.9. Define a Borel map $\varphi: \mathrm{SA}(H) \rightarrow X_{0}$ by $\varphi(A):=\left(d_{n}(A)\right)_{n=0}^{\infty}$. Since $H$ is infinite-dimensional, $\varphi(A) \in X_{0}$. Moreover, $A E_{\operatorname{dom}, u}^{\mathrm{SA}(H)} B$ if and only if $\varphi(A) E_{\Sigma} \varphi(B)$ by Theorem 2.4. Therefore $E_{\mathrm{dom}, u}^{\mathrm{SA}(H)} \leq\left._{B} E_{\Sigma}\right|_{X_{0}} \leq_{B} E_{\Sigma}$. To show $\left.E_{\Sigma}\right|_{X_{0}} \leq_{B} E_{\mathrm{dom}, u}^{\mathrm{SA}(H)}$, let

$$
X_{0, k}:=\left\{\left(a_{n}\right)_{n=0}^{\infty} \in X_{0} ; \sharp\left\{n \in \mathbb{N} \cup\{0\} ; a_{n}=\infty\right\}=k\right\}, \quad k \in \mathbb{N}^{*} \cup\{0\} .
$$

Note that each $X_{0, k}$ is a Borel subset of $X_{0}$ : it is enough to see that $\widetilde{X}_{0, k}:=\bigcup_{i=0}^{k} X_{0, i}$ is closed in $X$. But if $\alpha_{i}=\left(a_{n, i}\right)_{n=0}^{\infty} \in \widetilde{X}_{0, k}$ tends to $\alpha=\left(a_{n}\right)_{n=0}^{\infty} \in X_{0}$, then if $a_{n_{1}}=\cdots=$ $a_{n_{p}}=\infty\left(n_{1}<n_{2}<\cdots<n_{p}\right)$, then by assumption there exists $i_{0}$ such that for each $i \geq i_{0}$ $a_{i, n_{1}}=\cdots=a_{i, n_{p}}=\infty$, so $p \leq k$. Therefore $\alpha \in \widetilde{X}_{0, k}$, and $\widetilde{X}_{0, k}$ is closed.

Now define for each $k \in \mathbb{N}^{*} \cup\{0\}$ a Borel map $\psi_{k}: X_{0, k} \rightarrow \mathrm{SA}(H)$ by the following:

Case $k=0$.

Fix a CONS $\left\{\xi_{n}\right\}_{n=1}^{\infty}$ for $H$. For $\alpha=\left(a_{n}\right)_{n=0}^{\infty} \in X_{0,0}$, define

$$
\psi_{0}(\alpha):=\sum_{n=0}^{\infty}\left(2^{\frac{n}{2}}-1\right) e_{n}(\alpha)
$$


where the projection $e_{n, 0}(\alpha)$ is inductively defined as follows: $e_{0,0}(\alpha)$ is the projection onto $\operatorname{span}\left\{\xi_{1}, \cdots, \xi_{a_{0}}\right\}$ (if $a_{0} \geq 1$ ) and $e_{0,0}(\alpha)=0$ otherwise, and for $k \geq 0$,

$$
e_{k+1,0}(\alpha):=\text { projection onto } \operatorname{span}\left\{\xi_{a_{0}+\cdots+a_{k}+1}, \cdots \xi_{a_{0}+\cdots+a_{k}+a_{k+1}}\right\} \text { if } a_{k+1} \geq 1,
$$

and $e_{k+1,0}(\alpha):=0$ otherwise. Then it is easy to see that $\psi_{0}: X_{0,0} \rightarrow \mathrm{SA}(H)$ is continuous, and $T_{\psi_{0}(\alpha)}=\sum_{n=0}^{\infty} 2^{-n} e_{n, 0}(\alpha)$. In particular, the rank of the associated subspace for $T_{\psi_{0}(\alpha)}$ is $d_{n}\left(\psi_{0}(\alpha)\right)=a_{n}(n \geq 0)$.

Case $1 \leq k \leq \infty$.

Let $\alpha=\left(a_{n}\right)_{n=0}^{\infty} \in X_{0, k}$, and suppose that $a_{n_{1}}=\cdots=a_{n_{k}}=\infty\left(n_{1}<\cdots<n_{k}\right)$ (for $k=\infty$ case this means that $n_{1}<n_{2}<\cdots$ is an infinite sequence) and $a_{n}<\infty\left(n \notin\left\{n_{1}, \cdots, n_{k}\right\}\right)$. Fix another CONS $\left\{\eta_{n}, \zeta_{p, n} ; n \geq 1,1 \leq p \leq k\right\}$ for $H$, and define $\psi_{k}(\alpha) \in \operatorname{SA}(H)$ by

$$
\psi_{k}(\alpha):=\sum_{n=0}^{\infty}\left(2^{\frac{n}{2}}-1\right) e_{n, k}(\alpha)
$$

where the projection $e_{n, k}(\alpha)$ is defined as follows: define $\left(b_{n}\right)_{n=0}^{\infty} \in X_{0}$ inductively by

$$
b_{0}:=\left\{\begin{array}{cc}
a_{0} & \left(a_{0}<\infty\right) \\
0 & \left(a_{0}=\infty\right)
\end{array}, \quad b_{k+1}:=\left\{\begin{array}{cc}
b_{k}+a_{k+1} & \left(a_{k+1}<\infty\right) \\
b_{k} & \left(a_{k+1}=\infty\right)
\end{array}, \quad k \geq 0\right.\right.
$$

and then put $e_{0, k}(\alpha)=$ projection onto $\operatorname{span}\left\{\eta_{1}, \cdots, \eta_{b_{0}}\right\}$ if $a_{0}<\infty$, and $e_{0, k}(\alpha):=$ projection onto $\overline{\operatorname{span}}\left\{\zeta_{1, i}\right\}_{i=1}^{\infty}$ if $a_{0}=\infty$. For $n \geq 1$, put

$$
e_{n, k}(\alpha):=\left\{\begin{array}{cl}
0 & \left(a_{n}=0\right) \\
\text { projection onto } \operatorname{span}\left\{\eta_{b_{n-1}+1}, \cdots, \eta_{b_{n}}\right\} & \left(0<a_{n}<\infty\right) . \\
\text { projection onto } \operatorname{span}\left\{\zeta_{p, i}\right\}_{i=1}^{\infty} & \left(n=n_{p}\right)
\end{array}\right.
$$

Again $\psi_{k}: X_{0, k} \rightarrow \mathrm{SA}(H)$ is continuous, and $d_{n}\left(\psi_{k}(\alpha)\right)=a_{n}(n \geq 0)$.

Finally define $\psi: X_{0} \rightarrow \mathrm{SA}(H)$ by $\left.\psi\right|_{X_{0, k}}:=\psi_{k}$. Then since each $X_{0, k}$ is Borel and $\psi_{k}$ is continuous on $X_{0, k}, \psi$ is Borel. Moreover, since $d_{n}(\psi(\alpha))=a_{n}(n \geq 0)$ for every $\alpha=\left(a_{n}\right)_{n=0}^{\infty} \in X_{0}$, it follows that $\alpha E_{\Sigma} \beta \Leftrightarrow \psi(\alpha) E_{\text {dom }, u}^{\mathrm{SA}(H)} \psi(\beta)$ for $\alpha, \beta \in X_{0}$. This shows that $\left.E_{\Sigma}\right|_{X_{0}} \leq_{B} E_{\mathrm{dom}, u}^{\mathrm{SA}(H)}$. Therefore $\left.E_{\Sigma}\right|_{X_{0}} \sim_{B} E_{\mathrm{dom}, u}^{\mathrm{SA}(H)}$ holds.

Corollary 3.11. $E_{\mathrm{dom}, u}^{\mathrm{SA}(H)} \leq_{B} E_{\mathrm{dom}}^{\mathrm{SA}(H)}$ holds.

Proof. By Proposition 3.8. Theorems 3.1 and 3.2, it holds that $E_{\mathrm{dom}, u}^{\mathrm{SA}(H)} \leq_{B} E_{\ell^{\infty}}^{\mathbb{R}^{\mathbb{N}}} \sim_{c} E_{\mathrm{dom}}^{\mathrm{SA}(H)}$.

Remark 3.12. It is not clear whether $E_{\mathrm{dom}}^{\mathrm{SA}(H)} \leq_{B} E_{\mathrm{dom}, u}^{\mathrm{SA}(H)}$ holds.

\section{Generic $A$ has purely singular continuous spectrum $\mathbb{R}$}

In [AM14, Theorem $3.17(1)$ ], we have shown a genericity result that the set $\{A \in$ $\left.\mathrm{SA}(H) ; \sigma_{\text {ess }}(A)=\mathbb{R}\right\}$ is dense $G_{\delta}$ in $\mathrm{SA}(H)$. In this last section, we show that generic self-adjoint operators in fact have much more pathological spectral property:

Theorem 4.1. The set $\mathcal{G}:=\left\{A \in \mathrm{SA}(H) ; \sigma_{\mathrm{p}}(A)=\sigma_{\mathrm{ac}}(A)=\emptyset, \sigma_{\mathrm{sc}}(A)=\mathbb{R}\right\}$ is dense $G_{\delta}$ in $\mathrm{SA}(H)$. 
The proof relies on the surprising theorem of Simon (which he calls "Wonderland Theorem").

Definition 4.2. Sim95 Let $(X, d)$ be a metric space of self-adjoint operators on $H . X$ is called a regular metric space, if $d$ is complete and generates a topology stronger than or equal to SRT.

Theorem 4.3 (Simon's Wonderland Theorem). Let $(X, d)$ be a regular metric space of selfadjoint operators on $H$. Suppose that for some open interval $(a, b)$,

(1) $\{A \in X ; A$ has purely continuous spectrum on $(a, b)\}$ is dense in $X$.

(2) $\{A \in X ; A$ has purely singular spectrum on $(a, b)\}$ is dense in $X$.

(3) $\{A \in X ; A$ has $(a, b)$ in its spectrum $\}$ is dense in $X$.

Then $\left\{A \in X ;(a, b) \subset \sigma_{\mathrm{sc}}(A),(a, b) \cap \sigma_{\mathrm{p}}(A)=\emptyset,(a, b) \cap \sigma_{\mathrm{ac}}(A)=\emptyset\right\}$ is dense $G_{\delta}$ in $X$.

First we prove the density.

Proposition 4.4. The set $\left\{A \in \mathrm{SA}(H) ; \sigma_{\mathrm{p}}(A)=\sigma_{\mathrm{ac}}(A)=\emptyset\right\}$ is dense in $\mathrm{SA}(H)$.

Lemma 4.5. Let $H$ be an infinite-dimensional separable Hilbert space. There exists a sequence $\left\{A_{n}\right\}_{n=1}^{\infty} \subset \mathrm{SA}(H)$ with purely singular continuous spectrum, such that $A_{n} \stackrel{\mathrm{SRT}}{\rightarrow} 1_{H}$.

Proof. Let $\mu$ be a singular continuous probability measure on $\mathbb{R}$. We identify $H=L^{2}(\mathbb{R}, \mu)$, and define $A_{n}$ to be the multiplication by $f_{n}$, where $f_{n}(x):=\frac{1}{n} x+1 \quad(x \in \mathbb{R}, n \in \mathbb{N})$. Then each $A_{n}$ has purely singular continuous spectrum, and $A_{n} \stackrel{\text { SRT }}{\rightarrow} 1_{H}$ by Lebesgue Dominated Convergence Theorem.

Proof of Proposition 4.4. Let $A \in \mathrm{SA}(H)$ and let $\mathcal{V}$ be an SRT-open neighborhood of $A$. By Weyl-von Neumann Theorem, there exists $A_{0} \in \mathcal{V}$ of the form $A_{0}=\sum_{n=1}^{\infty} a_{n}\left\langle\xi_{n}, \cdot\right\rangle \xi_{n}$, where $\left\{a_{n}\right\}_{n=1}^{\infty} \subset \mathbb{R}$ and $\left\{\xi_{n}\right\}_{n=1}^{\infty}$ is an orthonormal basis for $H$. Let $e_{n}$ be the orthogonal projection of $H$ onto $\mathbb{C} \xi_{n}(n \in \mathbb{N})$. Let $k \in \mathbb{N}$. Choose a sequence of disjoint subsets $I_{1}^{(k)}, I_{2}^{(k)}, \cdots, I_{k}^{(k)}$ of $\mathbb{N} \backslash\{1,2, \cdots, k\}$ such that $\left|I_{1}^{(k)}\right|=\left|I_{2}^{(k)}\right|=\cdots=\left|I_{k}^{(k)}\right|=\infty$ and $\mathbb{N} \backslash\{1, \cdots, k\}=$ $\bigsqcup_{i=1}^{k} I_{i}^{(k)}$. Then for each $1 \leq i \leq k$, let $e_{i}^{(k)}$ be the projection of $H$ onto the closed linear span of $\left\{\xi_{m} ; m \in I_{i}^{(k)}\right\}$, which is of infinite-rank. Define a new operator $A_{k} \in \mathrm{SA}(H)$ by $A_{k}:=\sum_{n=1}^{k} a_{n} e_{n}+\sum_{n=1}^{k} a_{n} e_{n}^{(k)}$. Then $A_{k} \stackrel{k \rightarrow \infty}{\rightarrow} A_{0}$ (SRT), so that there exists $k_{0} \in \mathbb{N}$ such that $A_{k_{0}} \in \mathcal{V}$ holds. Now let $H_{i}\left(1 \leq i \leq k_{0}\right)$ be the range of $e_{i}+e_{i}^{\left(k_{0}\right)}$, which is infinite-dimensional. Thus by Lemma 4.5, we may find a sequence $\left\{A_{i, m}\right\}_{m=1}^{\infty} \subset \mathrm{SA}\left(H_{i}\right)$ with $\sigma_{\mathrm{p}}\left(A_{i, m}\right)=\sigma_{\mathrm{ac}}\left(A_{m, i}\right)=\emptyset(m \in \mathbb{N})$ such that $A_{i, m} \stackrel{m \rightarrow \infty}{\rightarrow} a_{i} 1_{H_{i}}$ (SRT) for each $1 \leq i \leq k_{0}$. Let $A_{m}:=\bigoplus_{i=1}^{k_{0}} A_{i, m} \in \mathrm{SA}(H)(m \in \mathbb{N})$. It follows that $A_{m} \stackrel{m \rightarrow \infty}{\rightarrow} A_{k_{0}}=\sum_{i=1}^{k_{0}} a_{i}\left(e_{i}+e_{i}^{\left(k_{0}\right)}\right) \in \mathcal{V}$ (SRT), so that there exists $m_{0} \in \mathbb{N}$ such that $A_{m_{0}} \in \mathcal{V}$. Since $\sigma_{\mathrm{p}}\left(A_{m_{0}}\right)=\sigma_{\mathrm{ac}}\left(A_{m_{0}}\right)=\emptyset$ and $\mathcal{V}$ is arbitrary, the claim follows.

Proof of Theorem 4 .1. For each $n \in \mathbb{N}$ define

$$
G_{n}:=\left\{A \in \mathrm{SA}(H) ; \sigma_{\mathrm{p}}(A) \cap(-n, n)=\sigma_{\mathrm{ac}}(A) \cap(-n, n)=\emptyset,(-n, n) \subset \sigma_{\mathrm{sc}}(A)\right\} .
$$

Since $\mathcal{G}=\bigcap_{n \in \mathbb{N}} G_{n}$, it suffices to show that each $G_{n}$ is dense $G_{\delta}$ in $\operatorname{SA}(H)$. We see that assumptions of Theorem 4.3 are satisfied for $X=\mathrm{SA}(H)$ with $(a, b)=(-n, n)$ :

(1) and (2): the sets

$$
\{A \in \mathrm{SA}(H) ; A \text { has purely continuous spectrum on }(-n, n)\}
$$


and

$$
\{A \in \mathrm{SA}(H) ; A \text { has purely singular spectrum on }(-n, n)\}
$$

are dense in $\mathrm{SA}(H)$, by Proposition 4.4 .

(3): By [AM14, Theorem $3.17(1)]$, the set $\mathrm{SA}_{\text {full }}(H)=\left\{A \in \mathrm{SA}(H) ; \sigma_{\text {ess }}(A)=\mathbb{R}\right\}$ is a dense $G_{\delta}$ subset of $\mathrm{SA}(H)$. In particular, $\{A \in \mathrm{SA}(H) ;(-n, n) \subset \sigma(A)\}$ is dense in $\mathrm{SA}(H)$.

Therefore By Theorem 4.3, $G_{n}$ is dense $G_{\delta}$ in $\mathrm{SA}(H)$ for every $n \in \mathbb{N}$, which finishes the proof.

\section{Acknowledgments}

The authors would like to thank the anonymous referee for numerous suggestions which improved the presentation of the paper. HA was supported by the Danish National Research Foundation through the Centre for Symmetry and Deformation (DNRF92). YM was supported by KAKENHI 26800055 and 26350231.

\section{References}

[AM14] H. Ando and Y. Matsuzawa, Weyl-von Neumann theorem and Borel complexity of unitary equivalence modulo compacts of self-adjoint operators, to appear in Proceedings of the Royal Society of Edinburgh, Section: A Mathematics.

[CN98] J. R. Chokski and M. G. Nadkarni, Genericity of certain classes of unitary and selfadjoint operators, Canad. Bull. Math. 41 (1998), 137-139.

[Dou66] R. G. Douglas, On majorization, factorization and range inclusion of operators in Hilbert space, Proc. Amer. Math. Soc. 17 (1966), 413-416.

[FW71] P. A. Fillmore and J. P. Williams, On operator ranges, Adv. Math. 7 (1971), 254-281.

[Gao09] S. Gao, Invariant descriptive set theory, CRC Press (2009).

[Hjo00] G. Hjorth, Classification and orbit equivalence relations, Mathematical Surveys and Monographs, 75, American Mathematical Society, Providence, RI (2000).

[Isr04] R. B. Israel, Some Generic Results in Mathematical Physics, Markov Processes and Related Fields 10 (2004), 517-521.

[Kec96] A. S. Kechris, Classical descriptive set theory, Springer-Verlag (1996).

[KL97] A. S. Kechris and A. Louveau, The classification of hypersmooth Borel equivalence relations, J. Amer. Math. Soc. 10 (1997), 215-242.

[LPS05] K. Latrach, J. Martin Paoli and P. Simonnet, Some facts from descriptive set theory concerning essential spectra and applications, Studia Math. 171 (2005), 207-225.

[Ros05] C. Rosendal, Cofinal families of Borel equivalence relations and quasiorders, J. Symbolic Logic 70 (2005), 1325-1340.

[RS81] M. Reed and B. Simon, Methods of modern mathematical physics, vol I: Functional Analysis, Academic Press (1981). 
[Sch10] K. Schmüdgen, Unbounded self-adjoint operators on Hilbert space, Graduate Texts in Mathematics 265, Springer-Verlag (2010).

[Sim95] B. Simon, Operators with singular continuous spectrum: I. General operators, Ann. Math. vol. 141, no. 1 (1995), 131-145.

Hiroshi Ando

Department of Mathematical Sciences,

University of Copenhagen

Universitetsparken 5

2100 Copenhagen $\varnothing$ Denmark

ando@math.ku.dk

http://andonuts.miraiserver.com/index.html

Yasumichi Matsuzawa

Department of Mathematics, Faculty of Education, Shinshu University

6-Ro, Nishi-nagano, Nagano, 380-8544, Japan

myasu@shinshu-u.ac.jp

https://sites.google.com/site/yasumichimatsuzawa/home 